\title{
Etiological and Symptomatological Differences Between Hyperactive and Hypoactive Delirium Subtypes
}

\author{
Marzanna Derkacz Jedynak, Marta Sudoł Malisz, Andrzej Brodziak* and Alicja Różyk Myrta \\ Institute of Medical Sciences, University of Applied Sciences, Nysa, Poland \\ *Corresponding author: Andrzej Brodziak, Institute of Medical Sciences, University of Applied Sciences, Nysa, Poland
}

\begin{abstract}
The processes of ageing and the related impairment of maintaining homeostasis, understood as the loss of adaptive abilities, lead to an increased susceptibility to developing delirium among the elderly. The pathophysiological process of delirium development is dependent on the presence of causative and predisposing factors; for example, neurotransmission process disorders (cholinergic, serotonin and dopaminergic regulation), resulting in metabolic inefficiency of the brain. The symptoms of delirium differ in duration and severity; there are 3 clinical subtypes of delirium: hypoactive, hyperactive and mixed. Moreover, subsequent evaluations using methodological tools have made it possible to distinguish an additional subtype of delirium - the no-motor subtype. Recognizing the delirium subtype is essential - it identifies high-risk patients, has an effect on the procedures, treatment and further prognosis. Medical personnel working with elderly patients ought to be well familiar with the predisposing factors, non-pharmacological procedures, treatment and prognosis of delirium; they also are required to know how to differentiate between each delirium subtype. Physicians should be aware that the hypoactive subtype of delirium indicates a much worse prognosis for patients.
\end{abstract}

Keywords: Delirium; Subtypes; Hypoactive; Hyperactive; Mixed; No - motor; Delirium Motor Subtype Scale DMSS-4

\section{Introduction}

The process of ageing, resulting in decreased abilities to maintain homeostasis, understood as the lack of capabilities to adapt, leads to an increased susceptibility to developing delirium among elderly patients [1]. The presence of triggering along with existing predisposing factors causes acute or subacute impairment of brain tissue metabolism, leading to specific brain failure. Delirium is a neuropsychiatric disorder and is characterized by changes in consciousness and impaired cognitive functions, especially attention, orientation and thought processes [2,3]. These symptoms differ in duration and severity and are the basis -after taking the coexistent behavioral disorders into consideration-to distinguish (according to the Lipowski model) 3 clinical subtypes of delirium: hypoactive, hyperactive and mixed [4]. The clinical subtypes are distinguished by psychomotor changes; subsequent evaluations by means of methodological tools now enable the identification of another subtype of delirium-the no-motor subtype $[5,6]$. Recognizing the delirium subtype is essential - it enables highrisk patients to be discerned, and has an effect on the procedures, treatment and further prognosis.

\section{Discussion}

The basic pathophysiology of delirium relies on the metabolic inefficiency of the brain, dependent on neurotransmission process disorders, such as: cholinergic, serotonin and dopaminergic regulation $[7,8]$. As a result of physiological changes (impaired brain flow, increased activity of proinflammatory cytokines, changed excitability of cholinergic receptors - in particular the receptors of the neocortex and hippocampus [9] that occur in the organisms of elderly patients, their intellectual abilities decline. Serotonin is responsible for the correct development of cognitive functions, wakefulness and state of mind, that is why serotonin deficiency is seen as the basic pathomechanism of developing delirium [10]. Another neurotransmitter which is taken into consideration and is crucial for the pathophysiological process is melatonin, whose increased activity is connected with the hypoactive form of delirium [11]. The clinical picture of delirium may manifest itself in the form of three different motor subtypes, each differentiated by the range of motor activities. 
Among the factors predisposing the development delirium, those recognised as the best predictors for all motor delirium subtypes, enabling medical personnel to prognose changes in the clinical state of the patient are impaired cognitive functions and visuospatial disorders, diagnosed before hospitalization or recognised during hospital admission [12]. The hyperactive or hypoactive subtypes of delirium are believed to have different etiologic background [13]. The hypoactive subtype is characterized by: sluggishness, significant impediment to contact with the patient, apathy, depression and somnolence. The patient with this clinical type is calm, acquiescent - and that is why delirium is frequently not diagnosed. The hypoactive subtype is commonly present in severe somatic diseases (metabolic dysfunctions - liver and kidney inefficiency), it exists along toxicosis, but also subdural hematoma or brain tumors.

This subtype is connected with more severe cognitive impairment - patients with hypoactive delirium have greater dysfunctions in perception, attention or recalling, but there is no significant difference in orientation or language functions between groups of patients with other delirium subtypes [13]. The hyperactive subtype of delirium usually consists in excitation, behavioral dysfunctions are highly expressed and psychotic symptoms are also present. The hyperactive subtype is characterized by: anxiety, hyperactivity, fast speech, irritability, manifestations of aggression and prowess. The occurrence of the hyperactive subtype of delirium causes accidental injuries (falls, fractures). This subtype is commonly connected with addiction to alcohol or psychoactive substances. Mixed delirium proceeds with changes (both increase and decrease) in the level of patient agitation, accompanied by disorganized thinking and very often resulting in a complete lack of contact with the patient. Even bed-ridden patients get excited, which is manifested by e.g. picking bedding, mindless manipulation of venous and urinary catheters, removing underwear or tearing diapers.

The incidence of the hyperactive subtype of delirium ranges from $9 \%$ to $31 \%$, whereas the hypoactive subtype varies from $19 \%$ to $72 \%$ [13]. The occurrence of hypoactive and mixed subtypes of delirium are responsible for longer hospitalization [14]. The fluctuation of symptoms is characteristic for delirium and may help indicate its subtypes. The daily fluctuation of symptoms in particular subtypes differ from one another. In the mixed and hyperactive subtypes of delirium there are greater ranges in the fluctuation of all the symptoms, and in the mixed subtype this fluctuation mainly concerns psychomotor activity [15]. Development of the motion subtype of delirium is dependent on the influence of external factors (for example the intake of cholinergic medicines) on individual features of an elderly patient and current predisposing factors.

However, it is not clear how other delirium features affect other motion subtypes [16]. Delirium research should consider the differences in motor subtypes, enabling the improvement of strategies for proceeding in clinically different states of delirium and improvement of the measures of symptom severity and the risk of death [17]. In everyday medical activities the delirium subtypes are evaluated by means of DMSS-4 (Delirium Motor Subtype Scale - version 4). The results obtained by means of DMSS-4 enable quick assessment of the clinical delirium subtypes and are highly compliant with the results of DMSS, a scale which has been used since 2008 and was subjected to meticulous validation. The latest version of this tool has been greatly simplified: it consists of 4 questions about the patient's behavior and psychomotor activity. According to the obtained results, which are short statements - "yes" or "no"- a specific subtype of delirium is discerned [6]. The clinical subtypes of delirium disclosed by DMSS-4 are more clearly determined, which can facilitate and improve proceeding with delirium patients and also bring benefits in learning the pathomechanisms of developing delirium [18].

\section{Conclusion}

Proceeding in delirium must include all the essential elements in the development of the disease. Medical personnel working with elderly patients ought to be well familiar with the delirium predisposing factors, non-pharmacological procedures, treatment and prognosis. In the case of a patient developing delirium, medical personnel should apply tools enabling them to discern between delirium subtypes, based on possible differences in the possible fluctuation of symptoms and the resulting clinical implications and further prognosis. Physicians should be aware that the hypoactive subtype of delirium, which manifests itself only by symptoms of hyperactivity (or alternating with these symptoms), indicates a much worse prognosis for these patients [17].

\section{References}

1. Oh ES, Fong TG, Hshieh TT, Inouye SK (2017) Delirium in Older Persons: Advances in Diagnosis and Treatment. JAMA 318(12): 1161-1174.

2. World Health Organization (1983) International Statistical Classification of Diseases and Health Related Problems. 10th revision, Geneva.

3. American Psychiatric Association (2013) Diagnostic and statistical manual of mental disorder, Vth edition. American Psychiatric Association, Washington, USA.

4. Lipowski Z (1987) Delirium (acute confusional states). JAMA258(13): 1789-1792.

5. Meagher D, Moran M, Raju B, Leonard M, Donnelly S, et al. (2008) A new data-based motor subtype schema for delirium. J Neuropsychiatry Clin Neurosci 20(2): 185-193.

6. Boettger S, Nunez DG, Meyer R, Richter A, Schubert M, et al. (2017) Brief assessment of delirium subtypes: Psychometric evaluation of the Delirium Motor Subtype Scale (DMSS)-4 in the intensive care setting. Palliat Support Care 15(5): 535-543.

7. Marcantonio ER, Rudolph JL, Culley D, Crosby G, Alsop D, et al. (2006) Serum biomarkers for delirium. J Gerontol 61A (12): 1281-1286.

8. Inouye SK, Ferrucci L (2006) Elucidating the pathophysiology of delirium and the interrelationship of delirium and dementia. J Gerontol Med Sci 61A (12): 1277-1280.

9. Tune LE, Egeli S (1999) Acetylcholine and delirium. Dement Geriatr Cogn Disord 10(5): 342-344. 
10. Flacker JM, Lipsitz LA (1999) Neural mechanisms of delirium: current hypotheses and evolving concepts. J Gerontol Biol Sci 54A (6): B239-B246.

11. Lewis MC, Barnett SR (2004) Postoperative delirium: the tryptophan dyregulation model. Med Hypotheses 63(3): 402-406.

12. Pasinska P, Kowalska K, Klimiec E, Wilk A, Szyper Maciejowska A, et al. (2018) Poststroke Delirium Clinical Motor Subtypes: The PRospective Observational POLIsh Study (PROPOLIS).

13. Kumar A, Bakhla A, Gupta S, Raju B, Prasad A (2015) Etiologic and Cognitive Differences in Hyperactive and Hypoactive Delirium. Prim Care Companion CNS Disord 17(6).

14. Lee A, Mu J, Chiu C, Gin, Underwood M, et al. (2017) Effect of motor subtypes of delirium in the intensive care unit on fast-track failure after cardiac surgery. J Thorac Cardiovasc Surg 155(1): 268-275.
15. Kim S, Kim J, Kim W, Kim E, Kang H. et al. (2018) Do the Phenotypes of Symptom Fluctuation Differ Among Motor Subtypes in Patients with Delirium? Pain Symptom Manage 56(5): 667-677.

16. FitzGerald J (2018) Delirium clinical motor subtypes: a narrative review of the literature and insights from neurobiology. Aging \& Mental Health 22(4): 431-443.

17. Avelino Silva T, Campora F, Curiati J, Jacob Filho W (2018) Prognostic effects of delirium motor subtypes in hospitalized older adults: A prospective cohort study. PLoS One13(1): e0191092.

18. Meagher D, Adamis D, Leonard M, Trzepacz P, Grover S, et al. (2014) Development of an abbreviated version of the delirium motor subtyping scale (DMSS-4). Int Psychogeriatr 26(4): 693-702.
To Submit Your Article Click Here:

This work is licensed under Creative Commons Attribution 4.0 License

DOI: $10.32474 / 0 J N B D .2019 .02 .000134$

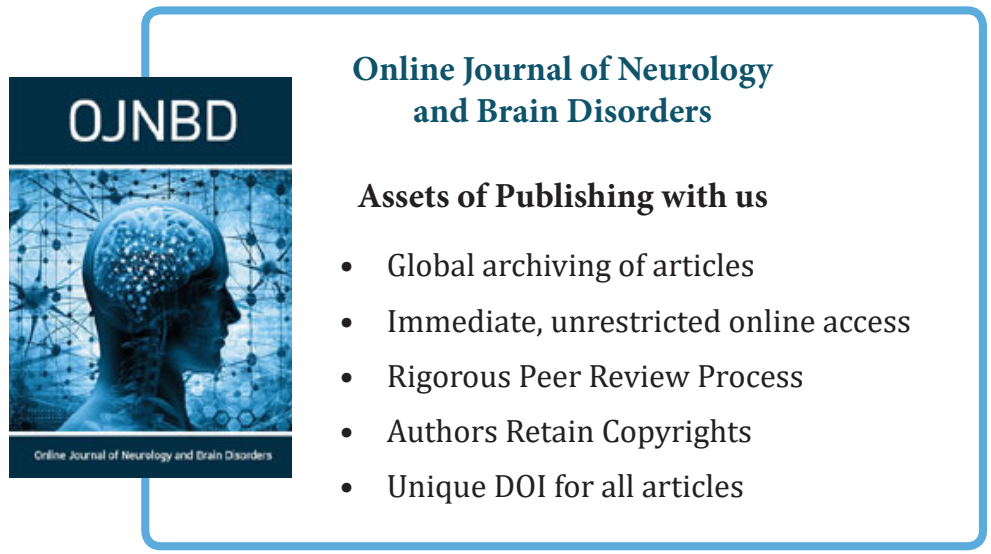

